\title{
NONAUTONOMOUS SUPERPOSITION OPERATORS IN THE SPACES OF FUNCTIONS OF BOUNDED VARIATION
}

\author{
Daria Bugajewska - Dariusz Bugajewski \\ Piotr Kasprzak — Piotr MaćKowiak
}

\begin{abstract}
The main goal of this paper is to give an answer to the main problem of the theory of nonautonomous superposition operators acting in the space of functions of bounded variation in the sense of Jordan. Namely, we give necessary and sufficient conditions which guarantee that nonautonomous superposition operators map that space into itself and are locally bounded. Moreover, special attention is drawn to nonautonomous superposition operators generated by locally bounded mappings as well as to superposition operators generated by functions with separable variables.
\end{abstract}

\section{Introduction}

The notion of a function of bounded variation is one of the basic notions of mathematical analysis. It was introduced by Camille Jordan (see [14]) in connection with his investigation on Fourier series.

It might seem that the problem of characterization of acting conditions for nonlinear superposition operators in the spaces of functions of bounded variation $\left({ }^{1}\right)$ has long been solved. It has been indeed, but only in the case of autonomous superposition operators (see [15]); the problem of stating necessary

2010 Mathematics Subject Classification. Primary: 47H30, 26A45; Secondary: 45G10.

Key words and phrases. Acting conditions; autonomous (nonautonomous) superposition operator; Lipschitz condition; locally bounded mapping; variation in the sense of Jordan.

$\left.{ }^{1}\right)$ In this paper we will be interested only in bounded variation in the sense of Jordan, and therefore we will refer to it simply as 'bounded variation'. 
and sufficient conditions for the nonautonomous superposition operator to map the space of functions of bounded variation into itself is still open.

In the monograph [4], on page 175 the authors write: "As already mentioned, no general results on the acting, boundedness, or continuity of the superposition operator $F$ are known in the nonautonomous case $f=f(t, u)$ (apart from trivial sufficient conditions, of course)."

On page 174 of that monograph the authors quote and prove the following result coming originally from Ljamin's paper [19].

Theorem 1.1. Assume that the function $f(t, \cdot)$ satisfies the Lipschitz condition on $\mathbb{R}$ uniformly in $t \in[0,1]$, and that the function $f(\cdot, u)$ is of bounded variation on the interval $[0,1]$ uniformly in $u \in \mathbb{R}$. Then the nonautonomous superposition operator $F$, generated by $f$, maps the space $\mathrm{BV}[0,1]$ into itself and is locally bounded, that is, it maps bounded sets into bounded ones.

(In the above theorem BV $[0,1]$ denotes the Banach space of all functions $x:[0,1] \rightarrow \mathbb{R}$ of bounded variation endowed with the norm $\|x\|_{\mathrm{BV}}=|x(0)|+\bigvee_{0}^{1} x$ for more details see Section 2.)

In the paper [6], Bugajewska formulated the conjecture that Theorem 1.1 might not be true. Let us also add that the proof of Ljamin's theorem presented in the survey article [3] is false. One can find the suitable examples confirming its falsity in the review by Bugajewski for ZblMATH (Zbl 1255.47059). The conjecture from the paper [6] was confirmed by Maćkowiak (see [22]) who presented the following counterexample to Theorem 1.1.

Example $1.2([22])$. Let the function $f:[0,1] \times \mathbb{R} \rightarrow \mathbb{R}$ be defined as follows:

$$
f(t, u)= \begin{cases}0 & \forall n \in\{2,3, \ldots\}: t \neq c_{n} \text { or } u \notin I_{n}, \\ \frac{1}{n}\left(1-\frac{\left|u-c_{n}\right|}{w_{n}}\right) & \exists n \in\{2,3, \ldots\}: t=c_{n} \text { and } u \in I_{n},\end{cases}
$$

where $c_{n}=1-1 / n, w_{n}=1 /(2 n)$ and $I_{n}=\left(c_{n}-w_{n}, c_{n}+w_{n}\right)$ for $n=2,3, \ldots$ For an arbitrary $t \in[0,1]$, the function $f(t, \cdot)$ satisfies the Lipschitz condition (uniformly in the second variable) with a Lipschitz constant not greater than 2. Moreover, $\bigvee_{0}^{1} f(\cdot, u) \leq 22$ for an arbitrary $u \in \mathbb{R}$. However, considering the functions $x(t)=t$ and $g(t)=f(t, x(t))$ for $t \in[0,1]$, one can easily be convinced that the nonautonomous superposition operator, generated by the function $f$, does not map the space BV $[0,1]$ into itself.

In the introduction to the recently published monograph $[2$, p. 6$]$, the authors stated three fundamental open problems of the theory of nonlinear superposition operators in the space of functions of bounded variation. The first problem 
mentioned there concerns both necessary and sufficient conditions which would imply that the nonautonomous superposition operator maps the space under consideration into itself. Thus we can say, roughly speaking, that the theory of nonautonomous superposition operators in the space of functions of bounded variation is still in its initial point.

The main purpose of this article is to provide an answer to the problem concerning acting conditions. Let us emphasize that the proof of sufficiency of our conditions stated in the main result (see Theorem 3.8) is straightforward, while the proof of their necessity is non-trivial and tricky (see also Theorem 4.4 for the case of nonlinear superposition operators generated by locally bounded functions).

Let us also add that the third problem stated in the mentioned monograph concerning local boundedness of a nonautonomous superposition operator was solved in the paper [17].

Let us emphasize that the theory of nonlinear superposition operators in the spaces of functions of bounded variation is closely connected with the theory of solutions to nonlinear equations in these classes of functions (see e.g. [7], [8], [13]). The study of so-called BV-solutions seems to be interesting for at least a few reasons. First, let us draw attention to the fact that solutions to the Cauchy problem for the equation of first order, defined on a compact interval in $\mathbb{R}$, the existence of which is guaranteed by the classical Peano theorem, are functions of bounded variation (at least locally). This property is preserved, if one considers solutions to this equation, the existence of which follows from the Carathéodory theorem (see [10, Theorem 1.1]). Second, solutions to many equations which describe concrete physical phenomena are functions of (local) bounded variation. As examples, we could mention here equations describing the amplitude of forced vibrations of a string, which appear in engineering (see [23]), or Volterra integral equations modelling population dynamics under constant harvesting (see e.g. [5]). Applications of functions of bounded variation in economics to describe dynamic choices of hyperbolic consumers (see e.g. [12]) are also worth mentioning.

The motivation for the study of solutions to nonlinear integral equations in the class of functions of bounded variation comes also from the theory of nonabsolute convergent integrals. Namely, it is well-known that if $h:[0,1] \rightarrow \mathbb{R}$ is a function integrable in the Denjoy-Perron sense (or, equivalently, in the Henstock-Kurzweil sense), then $h \varphi$ is also integrable in that sense whenever $\varphi:[0,1] \rightarrow \mathbb{R}$ is a function of bounded variation (see [9]).

Finally, let us mention that certain functions of bounded variation also possess essential applications, for example, in the geometric measure theory (see e.g. $[21],[1],[11]$ ) or in image processing to recovering images (see e.g. [18]). 
The structure of this paper is as follows. In Section 2 we fix notation used in this paper and we recall a few basic results concerning nonlinear superposition operators acting in the space of functions of bounded variation. Section 3 contains, among others, the main result of this paper, namely Theorem 3.8, which gives necessary and sufficient conditions guaranteeing that the nonautonomous superposition operator maps the space of functions of bounded variation into itself and its local boundedness. In Section 4 we examine nonautonomous superposition operators generated by locally bounded functions. A necessary and sufficient acting condition for such superposition operators is stated in Theorem 4.4. Finally, Section 5 contains exhaustive study of nonlinear superposition operators which are generated by functions with separable variables.

\section{Preliminaries}

Notation 2.1. The closed ball with center at $x$ and radius $r \in(0,+\infty)$ in a normed space $X$ will be denoted by $B_{X}(x, r)$. For simplicity, instead of $B_{\mathbb{R}}(x, r)$ we will obviously write $[x-r, x+r]$. By BV $[a, b]$ we will denote the Banach space of all real-valued functions of bounded variation defined on $[a, b]$, endowed with the norm $\|x\|_{\mathrm{BV}}:=|x(a)|+\bigvee_{a}^{b} x$; here the symbol $\bigvee_{a}^{b} x$ denotes the Jordan variation of the function $x:[a, b] \rightarrow \mathbb{R}$, that is,

$$
\bigvee_{a}^{b} x=\sup _{\pi} \sum_{i=1}^{n}\left|x\left(t_{i}\right)-x\left(t_{i-1}\right)\right|
$$

where the supremum is taken over all finite partitions $\pi: a=t_{0}<t_{1}<\ldots<$ $t_{n}=b$ of the interval $[a, b]$. Moreover, the set of points of discontinuity of a function $f$ will be denoted by $D_{f}$. If $u$ is an arbitrary real number, then by $x_{u}$ we will denote the constant function attaining only the value $u$, that is, $x_{u}(t)=u$ for every $t$ in the domain of $x_{u}$.

The necessary and sufficient acting condition for the autonomous superposition operators are fully described by the following Josephy's

Theorem 2.2 ([15], [4, Theorem 6.13]). Suppose that $F$ is an autonomous superposition operator generated by a function $f: \mathbb{R} \rightarrow \mathbb{R}$. The superposition operator $F$ maps the space $\mathrm{BV}[0,1]$ into itself if and only if the function $f$ satisfies a local Lipschitz condition, that is, for every $r>0$ there exists a number $L_{r} \geq 0$ such that $|f(u)-f(w)| \leq L_{r}|u-w|$, whenever $u, w \in[-r, r]$.

In this paper, by locally bounded mappings we will understand the mappings defined as follows. 
Definition 2.3. Let $X$ be a normed space. A mapping $F: X \rightarrow X$ is said to be locally bounded if for each $r>0$ there exists $R>0$ such that

$$
F\left(B_{X}(0, r)\right) \subset B_{X}(0, R) .
$$

For the case of nonautonomous superposition operators in the space of functions of bounded variation, the sufficient acting conditions are given in the following

TheOrem 2.4 ([6, Theorem 1]). Let the function $f:[0,1] \times \mathbb{R} \rightarrow \mathbb{R}$ satisfy the following conditions:

(a) $f$ satisfies a Lipschitz condition on $\mathbb{R}$ uniformly in $t \in[0,1]$;

(b) there exists a constant $M>0$ such that for arbitrary real numbers $u_{0}, \ldots, u_{n-1}$ and an arbitrary finite partition $0=t_{0}<t_{1}<\ldots<t_{n}=1$ of $[0,1]$ the following inequality holds:

$$
\sum_{i=1}^{n}\left|f\left(t_{i}, u_{i-1}\right)-f\left(t_{i-1}, u_{i-1}\right)\right| \leq M
$$

Then the nonautonomous superposition operator $F$, generated by $f$, maps the space $\mathrm{BV}[0,1]$ into itself and is locally bounded.

The above quoted result gave us a clue what kind of conditions might be necessary in the situation under consideration.

REMARK 2.5. Clearly, the conclusions of Theorems 2.2 and 2.4 remain true, if we replace the space $\mathrm{BV}[0,1]$ with the space $\mathrm{BV}[a, b]$ for arbitrary $a, b \in \mathbb{R}$ such that $a<b$.

\section{Nonautonomous superposition operators - a general case}

The first result of this section is a simple refinement of Theorem 2.4.

THEOREM 3.1. Let the function $f:[0,1] \times \mathbb{R} \rightarrow \mathbb{R}$ satisfy the following conditions:

(a) $f$ satisfies a local Lipschitz condition on $\mathbb{R}$ uniformly in $t \in[0,1]$;

(b) for every $r>0$ there exists a constant $M_{r}>0$ such that for every $n \in \mathbb{N}$, every partition $0=t_{0}<\ldots<t_{n}=1$ of $[0,1]$ and every $u_{0}, \ldots, u_{n-1} \in$ $[-r, r]$ the following implication holds:

$$
\sum_{i=1}^{n-1}\left|u_{i}-u_{i-1}\right| \leq r \quad \Rightarrow \quad \sum_{i=1}^{n}\left|f\left(t_{i}, u_{i-1}\right)-f\left(t_{i-1}, u_{i-1}\right)\right| \leq M_{r} .
$$

Then the superposition operator $F$, generated by $f$, maps the space $\mathrm{BV}[0,1]$ into itself and is locally bounded. 
Proof. Given $x \in \mathrm{BV}[0,1]$, let $r>0$ be such that $\|x\|_{\mathrm{BV}} \leq r$. Moreover, let $L_{r}$ denote the (uniform) Lipschitz constant corresponding to the function

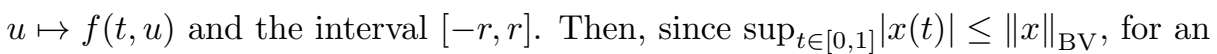
arbitrary finite partition $0=t_{0}<t_{1}<\ldots<t_{n}=1$ we have

$$
\begin{aligned}
& \sum_{i=1}^{n}\left|f\left(t_{i}, x\left(t_{i}\right)\right)-f\left(t_{i-1}, x\left(t_{i-1}\right)\right)\right| \\
& \leq \sum_{i=1}^{n}\left|f\left(t_{i}, x\left(t_{i}\right)\right)-f\left(t_{i}, x\left(t_{i-1}\right)\right)\right|+\sum_{i=1}^{n}\left|f\left(t_{i}, x\left(t_{i-1}\right)\right)-f\left(t_{i-1}, x\left(t_{i-1}\right)\right)\right| \\
& \leq L_{r} \sum_{i=1}^{n}\left|x\left(t_{i}\right)-x\left(t_{i-1}\right)\right|+\sum_{i=1}^{n}\left|f\left(t_{i}, x\left(t_{i-1}\right)\right)-f\left(t_{i-1}, x\left(t_{i-1}\right)\right)\right| .
\end{aligned}
$$

Hence

$$
\bigvee_{0}^{1} F(x) \leq L_{r} \bigvee_{0}^{1} x+M_{r}
$$

and therefore $F(x) \in \mathrm{BV}[0,1]$. Furthermore, we have

$$
\|F(x)\|_{\mathrm{BV}} \leq|f(0, x(0))|+L_{r} \bigvee_{0}^{1} x+M_{r} \leq|f(0,0)|+r L_{r}+M_{r},
$$

which shows that $F$ is locally bounded with $R:=|f(0,0)|+r L_{r}+M_{r}$.

The following example shows that the above result is an essential improvement of Theorem 2.4.

Example 3.2. Let us consider the function $f:[0,1] \times \mathbb{R} \rightarrow \mathbb{R}$ defined by the following formula:

$$
f(t, u)= \begin{cases}0 & \text { if } t \neq 1 / n \text { and } u \in \mathbb{R}, \\ 0 & \text { if } t=1 / n \text { and } u<n-1, \\ 1 & \text { if } t=1 / n \text { and } u \geq n, \\ u-(n-1) & \text { if } t=1 / n \text { and } n-1 \leq u<n,\end{cases}
$$

where $n \in \mathbb{N}$. Let us observe that for any $t \in[0,1]$ the function $u \mapsto f(t, u)$ satisfies a Lipschitz condition with the constant at most 1. Furthermore, $f$ does not satisfy condition (b) of Theorem 2.4. Indeed, for every positive integer $n \geq 2$ let

$$
u_{0}:=0, \quad u_{1}:=n-1, \quad \ldots, \quad u_{i}:=n-i, \quad \ldots, \quad u_{n-1}:=1
$$

and

$$
t_{0}:=0, \quad t_{1}=\frac{1}{n}, \quad \ldots, \quad t_{i}:=\frac{1}{n-i+1}, \quad \ldots, \quad t_{n}:=1 .
$$


Then

$$
\begin{aligned}
& \sum_{i=1}^{n}\left|f\left(t_{i}, u_{i-1}\right)-f\left(t_{i-1}, u_{i-1}\right)\right|=\left|f\left(\frac{1}{n}, 0\right)-f(0,0)\right| \\
& \quad+\sum_{i=2}^{n}\left|f\left(\frac{1}{n-i+1}, n-i+1\right)-f\left(\frac{1}{n-i+2}, n-i+1\right)\right| \geq n-1 .
\end{aligned}
$$

On the other hand, the function $f$ satisfies condition (b) of Theorem 3.1, since for an arbitrary positive number $r>0$, in view of the fact that in each region $[0,1] \times[-r, r]$ the function $f$ vanishes everywhere except at the set consisting of a finite number of vertical line segments, it suffices to take $M_{r}:=2[r]+1$, where $[r]$ denotes the integer part of the number $r$.

Proposition 3.3. Suppose that the function $f:[0,1] \times \mathbb{R} \rightarrow \mathbb{R}$ satisfies assumption (a) of Theorem 3.1. If the autonomous superposition operator F, generated by $f$, maps the space $\mathrm{BV}[0,1]$ into itself and is locally bounded, then the function $f$ satisfies condition (b) of Theorem 3.1 .

Proof. Given a number $r>0$, let $R>0$ be such that $F\left(B_{\mathrm{BV}}(0,2 r)\right) \subset$ $B_{\mathrm{BV}}(0, R)$. Moreover, let $L_{r}$ denote the (uniform) Lipschitz constant corresponding to the function $u \mapsto f(t, u)$ and the interval $[-r, r]$. Set $M_{r}:=r L_{r}+R$ and consider an arbitrary finite partition $0=t_{0}<t_{1}<\ldots<t_{n}=1$ of $[0,1]$ and an arbitrary finite collection of real numbers $u_{0}, \ldots, u_{n-1}$ such that $\sum_{i=1}^{n-1}\left|u_{i}-u_{i-1}\right| \leq r$. We infer that there exists a function $x \in \mathrm{BV}[0,1]$ such that $x\left(t_{i}\right)=u_{i}$ for $i=0, \ldots, n-1$ and $\bigvee_{0}^{1} x \leq r$. Indeed, it suffices to consider the function which is linear on each of the intervals $\left[t_{i-1}, t_{i}\right]$ for $i=0, \ldots, n-1$ and is parallel to the horizontal axis on the interval $\left[t_{n-1}, t_{n}\right]$ (cf. the proof of Theorem 3.8). Then we have

$$
\begin{aligned}
& \sum_{i=1}^{n}\left|f\left(t_{i}, u_{i-1}\right)-f\left(t_{i-1}, u_{i-1}\right)\right|=\sum_{i=1}^{n}\left|f\left(t_{i}, x\left(t_{i-1}\right)\right)-f\left(t_{i-1}, x\left(t_{i-1}\right)\right)\right| \\
& \quad \leq \sum_{i=1}^{n}\left|f\left(t_{i}, x\left(t_{i-1}\right)\right)-f\left(t_{i}, x\left(t_{i}\right)\right)\right|+\sum_{i=1}^{n}\left|f\left(t_{i}, x\left(t_{i}\right)\right)-f\left(t_{i-1}, x\left(t_{i-1}\right)\right)\right| \\
& \quad \leq L_{r} \sum_{i=1}^{n}\left|x\left(t_{i-1}\right)-x\left(t_{i}\right)\right|+\bigvee_{0}^{1} F(x) \leq r L_{r}+R=M_{r} .
\end{aligned}
$$

This ends the proof.

EXAMPLE 3.4. Let $F$ be the nonautonomous superposition operator generated by a function $f:[0,1] \times \mathbb{R} \rightarrow \mathbb{R}$. Let us emphasize that the fact that $F$ maps the space BV $[0,1]$ into itself does not have to imply that $F$ is locally bounded. 
Indeed, let $f:[0,1] \times \mathbb{R} \rightarrow \mathbb{R}$ be defined by the formula

$$
f(t, u)= \begin{cases}0 & \text { if } t \neq 0 \text { or } u \leq 0 \\ u^{-1} & \text { otherwise }\end{cases}
$$

Furthermore, for every $n \in \mathbb{N}$, let

$$
x_{n}(t)= \begin{cases}n^{-1} & \text { if } t=0, \\ 0 & \text { if } t \in(0,1] .\end{cases}
$$

Clearly, the superposition operator $F$, generated by the function $f$, maps $\operatorname{BV}[0,1]$ into itself. However, $\left\|F\left(x_{n}\right)\right\|_{\mathrm{BV}}=2 n$, while $\left\|x_{n}\right\|_{\mathrm{BV}}=2 n^{-1}$ for $n \in \mathbb{N}$.

Actually, the conclusion of the above remark follows from a more general result, namely from the following

Proposition 3.5. Suppose that a function $f:[0,1] \times \mathbb{R} \rightarrow \mathbb{R}$ generates the nonautonomous superposition operator $F$ which maps the space $\mathrm{BV}[0,1]$ into itself. If the function $f$ is not locally bounded, then also the operator $F$ is not locally bounded.

Proof. Since the function $f$ is not locally bounded, there exist a constant $r>0$ and a sequence $\left(t_{n}, u_{n}\right)_{n \in \mathbb{N}}$ in $[0,1] \times[-r, r]$ such that $\lim _{n \rightarrow \infty}\left|f\left(t_{n}, u_{n}\right)\right|=$ $+\infty$. Clearly, without loss of generality, we may assume that the sequence $\left(t_{n}\right)_{n \in \mathbb{N}}$ converges to a point $t_{0} \in[0,1]$. For each $n \in \mathbb{N}$ define a function $x_{n}:[0,1] \rightarrow \mathbb{R}$ by

Notice that

$$
x_{n}(t)= \begin{cases}u_{n} & \text { if } t=t_{n} \\ 0 & \text { otherwise }\end{cases}
$$

$$
\left\|x_{n}\right\|_{\mathrm{BV}}=\left|x_{n}(0)\right|+\bigvee_{0}^{1} x_{n} \leq 3 r \quad \text { for every } n \in \mathbb{N},
$$

and therefore, in view of the assumption, $F\left(x_{n}\right) \in \mathrm{BV}[0,1]$ for every $n \in \mathbb{N}$. On the other hand, for sufficiently large $n \in \mathbb{N}$, we have

$$
\begin{aligned}
\left|f\left(t_{n}, u_{n}\right)\right|-|f(s, 0)| & \leq\left|f\left(t_{n}, u_{n}\right)-f(s, 0)\right| \\
& =\left|f\left(t_{n}, x_{n}\left(t_{n}\right)\right)-f\left(s, x_{n}(s)\right)\right| \leq \bigvee_{0}^{1} F\left(x_{n}\right),
\end{aligned}
$$

where $s$ is an arbitrary (but fixed) point in $[0,1]$ distinct from $t_{0}$. Hence

$$
\lim _{n \rightarrow \infty} \bigvee_{0}^{1} F\left(x_{n}\right)=+\infty
$$

The fact that the nonautonomous superposition operator maps the space $\mathrm{BV}[0,1]$ into itself implies also the property stated as 
Proposition 3.6. If the nonautonomous superposition operator $F$, generated by a function $f:[0,1] \times \mathbb{R} \rightarrow \mathbb{R}$, maps the space $\mathrm{BV}[0,1]$ into itself, then for every $r>0$ the set $T_{r}:=\left\{t \in[0,1]: \sup _{u \in[-r, r]}|f(t, u)|=+\infty\right\}$ is finite.

Proof. Let us assume that the superposition operator $F$ maps the space $\mathrm{BV}[0,1]$ into itself and suppose that there exists $r>0$ such that the set $T_{r}$ is (at least) denumerable $\left({ }^{2}\right)$. Thus, we can find a sequence $\left(t_{n}, u_{n}\right)_{n \in \mathbb{N}}$ in $[0,1] \times[-r, r]$ such that

$$
\left|f\left(t_{n+1}, u_{n+1}\right)\right| \geq\left|f\left(t_{n}, u_{n}\right)\right|+1 \quad \text { for } n \in \mathbb{N} .
$$

Let us observe that, without loss of generality, we may assume that:

(a) the sequences $\left(t_{n}\right)_{n \in \mathbb{N}}$ and $\left(u_{n}\right)_{n \in \mathbb{N}}$ are monotone;

(b) $t_{n} \rightarrow t_{0}$ and $u_{n} \rightarrow u_{0}$, as $n \rightarrow+\infty$, for some $t_{0} \in[0,1]$ and $u_{0} \in[-r, r]$;

(c) $t_{n} \neq t_{m}$, if $n \neq m$.

To prove the above claim note that, in view of the denumerability of the set $T_{r}$, the sequence $\left(t_{n}\right)_{n \in \mathbb{N}}$ can be chosen in such a way that $t_{n} \neq t_{m}$, if $n \neq m$. Furthermore, since $\left(t_{n}, u_{n}\right) \in[0,1] \times[-r, r]$ for every $n \in \mathbb{N}$, the sequence $\left(t_{n}, u_{n}\right)_{n \in \mathbb{N}}$ admits a convergent subsequence $\left(t_{n_{k}}, u_{n_{k}}\right)_{k \in \mathbb{N}}$, that is, there exist points $t_{0} \in[0,1]$ and $u_{0} \in[-r, r]$ such that $t_{n_{k}} \rightarrow t_{0}$ and $u_{n_{k}} \rightarrow u_{0}$ as $k \rightarrow+\infty$. Besides, from (3.2) it follows that

$$
\left|f\left(t_{m}, u_{m}\right)\right| \geq\left|f\left(t_{n}, u_{n}\right)\right|+1 \text { for } m>n,
$$

and thus to prove the claim, it now suffices to take any monotone subsequences $\left(t_{n_{k_{l}}}\right)_{l \in \mathbb{N}}$ of $\left(t_{n_{k}}\right)_{k \in \mathbb{N}}$ and $\left(u_{n_{k_{l}}}\right)_{l \in \mathbb{N}}$ of $\left(u_{n_{k}}\right)_{k \in \mathbb{N}}$, respectively.

Now, let $x:[0,1] \rightarrow \mathbb{R}$ be a function given by the following formula:

$$
x(t)= \begin{cases}u_{n} & \text { if } t=t_{n} \text { for } n \in \mathbb{N}, \\ u_{0} & \text { if } t=t_{0}, \\ \operatorname{linear} & \text { on }\left(\min \left\{t_{n}, t_{n+1}\right\}, \max \left\{t_{n}, t_{n+1}\right\}\right) \text { for } n \in \mathbb{N}, \\ x\left(\sup _{n \in \mathbb{N}} t_{n}\right) & \text { if } t \in\left(\sup _{n \in \mathbb{N}} t_{n}, 1\right], \\ x\left(\inf _{n \in \mathbb{N}} t_{n}\right) & \text { if } t \in\left[0, \inf _{n \in \mathbb{N}} t_{n}\right) .\end{cases}
$$

It can be shown that the function $x$ is monotone and hence $x \in \mathrm{BV}[0,1]$. On the other hand, for every positive integer $n \geq 2$, we have

$$
\begin{aligned}
\bigvee_{0}^{1} F(x) & \geq \sum_{i=1}^{n-1}\left|F(x)\left(t_{i+1}\right)-F(x)\left(t_{i}\right)\right| \\
& \geq \sum_{i=1}^{n-1}\left(\left|f\left(t_{i+1}, x\left(t_{i+1}\right)\right)\right|-\left|f\left(t_{i}, x\left(t_{i}\right)\right)\right|\right) \geq n-1,
\end{aligned}
$$

$\left({ }^{2}\right)$ Recall that a set is called denumerable if it is infinite and countable. 
which proves that $F(x) \notin \mathrm{BV}[0,1]$. This contradicts our assumption. Therefore, the set $T_{r}$ is finite.

The following result states, in particular, that thinking about necessary acting conditions for the nonautonomous superposition operator in the space $\mathrm{BV}[0,1]$, one cannot say anything about the behaviour of the generator of that operator with respect to the second variable.

THEOREM 3.7. Let $F$ be a nonautonomous superposition operator, generated by a function $f:[0,1] \times \mathbb{R} \rightarrow \mathbb{R}$, which maps the space $\mathrm{BV}[0,1]$ into itself. Then for every $u \in \mathbb{R}$ the function $t \mapsto f(t, u)$ is of bounded variation. Furthermore, in general, nothing can be said about the function $u \mapsto f(t, u)$, where $t \in[0,1]$ is fixed.

Proof. For every $u \in \mathbb{R}$, in view of the assumption, $F\left(x_{u}\right) \in \mathrm{BV}[0,1]$, that is, the function $t \mapsto f(t, u)$ is of bounded variation.

To show the second claim, let us consider the function $f:[0,1] \times \mathbb{R} \rightarrow \mathbb{R}$ given by the formula $f(t, u)=h(t) g(u)$, where $g: \mathbb{R} \rightarrow \mathbb{R}$ is an arbitrary function and $h:[0,1] \rightarrow \mathbb{R}$ is defined by

$$
h(t)= \begin{cases}0 & \text { if } t \in(0,1], \\ 1 & \text { if } t=0 .\end{cases}
$$

Then the nonautonomous superposition operator $F$, generated by the function $f$, is given by

$$
F(x)(t)= \begin{cases}0 & \text { if } t \in(0,1], \\ g(x(0)) & \text { if } t=0,\end{cases}
$$

where $x \in \mathrm{BV}[0,1]$, and hence it maps the space $\mathrm{BV}[0,1]$ into itself.

Now, we are going to prove the main result of this paper concerning necessary and sufficient conditions for the inclusion $F(\mathrm{BV}[0,1]) \subset \mathrm{BV}[0,1]$ and the local boundedness of the nonautonomous superposition operator $F$.

ThEOREM 3.8. Suppose that $f:[0,1] \times \mathbb{R} \rightarrow \mathbb{R}$ is a given function. The following conditions are equivalent:

(a) the nonautonomous superposition operator $F$, generated by $f$, maps the space $\mathrm{BV}[0,1]$ into itself and is locally bounded;

(b) for every $r>0$ there exists a constant $M_{r}>0$ such that for every $k \in \mathbb{N}$, every finite partition $0=t_{0}<\ldots<t_{k}=1$ of the interval $[0,1]$ and every finite sequence $u_{0}, \ldots, u_{k} \in[-r, r]$ with $\sum_{i=1}^{k}\left|u_{i}-u_{i-1}\right| \leq r$, the following inequalities hold

$\sum_{i=1}^{k}\left|f\left(t_{i}, u_{i}\right)-f\left(t_{i-1}, u_{i}\right)\right| \leq M_{r} \quad$ and $\quad \sum_{i=1}^{k}\left|f\left(t_{i-1}, u_{i}\right)-f\left(t_{i-1}, u_{i-1}\right)\right| \leq M_{r}$. 
Proof. $(\mathrm{b}) \Rightarrow(\mathrm{a})$. Let $x \in \mathrm{BV}[0,1]$ be such that $\|x\|_{\mathrm{BV}} \leq r$. Moreover, let $M_{r}$ be a constant for which condition (b) is satisfied and let $0=t_{0}<\ldots<$ $t_{k}=1$ be any finite partition of the interval $[0,1]$. Then, in view of the triangle inequality, we have

$$
\sum_{i=1}^{k}\left|f\left(t_{i}, x\left(t_{i}\right)\right)-f\left(t_{i-1}, x\left(t_{i-1}\right)\right)\right| \leq 2 M_{r} .
$$

The above inequality and the fact that $f$ is locally bounded (which is implied by (b)) ensure that $F$ is locally bounded.

$(\mathrm{a}) \Rightarrow(\mathrm{b})$. Suppose now that the superposition operator $F$ satisfies condition (a) and that there exists $r>0$ such that for every $n \in \mathbb{N}$ there exist a finite partition $0=t_{0}^{n}<\ldots<t_{k_{n}}^{n}=1$ of $[0,1]$ and a finite sequence $u_{0}^{n}, \ldots, u_{k_{n}}^{n} \in[-r, r]$ with $\sum_{i=1}^{k_{n}}\left|u_{i}^{n}-u_{i-1}^{n}\right| \leq r$ such that

$$
\begin{aligned}
& \text { either } \quad \sum_{i=1}^{k_{n}}\left|f\left(t_{i}^{n}, u_{i}^{n}\right)-f\left(t_{i-1}^{n}, u_{i}^{n}\right)\right|>n \\
& \quad \text { or } \quad \sum_{i=1}^{k_{n}}\left|f\left(t_{i-1}^{n}, u_{i}^{n}\right)-f\left(t_{i-1}^{n}, u_{i-1}^{n}\right)\right|>n .
\end{aligned}
$$

For every $n \in \mathbb{N}$ define auxiliary functions $\xi^{n}:[0,1] \rightarrow \mathbb{R}$ by

$$
\xi^{n}(t)= \begin{cases}u_{i}^{n} & \text { if } t=t_{i}^{n} \text { for some } i \in\left\{0,1, \ldots, k_{n}\right\}, \\ \frac{t_{i+1}^{n}-t}{t_{i+1}^{n}-t_{i}^{n}} u_{i}^{n}+\frac{t-t_{i}^{n}}{t_{i+1}^{n}-t_{i}^{n}} u_{i+1}^{n} & \text { if } t \in\left(t_{i}^{n}, t_{i+1}^{n}\right), \\ & \text { for some } i \in\left\{0, \ldots, k_{n}-1\right\} .\end{cases}
$$

Clearly,

$$
\bigvee_{0}^{1} \xi^{n}=\sum_{i=1}^{k_{n}}\left|\xi^{n}\left(t_{i}^{n}\right)-\xi^{n}\left(t_{i-1}^{n}\right)\right|=\sum_{i=1}^{k_{n}}\left|u_{i}^{n}-u_{i-1}^{n}\right| \leq r
$$

and since the superposition operator $F$ is locally bounded, there exists a constant $R>0$ (corresponding to $2 r$ ) such that $\sup _{n \in \mathbb{N}}\left\|F\left(\xi^{n}\right)\right\|_{\mathrm{BV}} \leq R$. In particular, for every $n \in \mathbb{N}$ we have

$$
\begin{aligned}
& \sum_{i=1}^{k_{n}}\left|f\left(t_{i}^{n}, u_{i}^{n}\right)-f\left(t_{i-1}^{n}, u_{i}^{n}\right)\right| \\
& \quad \leq \sum_{i=1}^{k_{n}}\left|f\left(t_{i}^{n}, u_{i}^{n}\right)-f\left(t_{i-1}^{n}, u_{i-1}^{n}\right)\right|+\sum_{i=1}^{k_{n}}\left|f\left(t_{i-1}^{n}, u_{i-1}^{n}\right)-f\left(t_{i-1}^{n}, u_{i}^{n}\right)\right| \\
& \quad \leq \sup _{n \in \mathbb{N}} \bigvee_{0}^{1} F\left(\xi^{n}\right)+\sum_{i=1}^{k_{n}}\left|f\left(t_{i-1}^{n}, u_{i-1}^{n}\right)-f\left(t_{i-1}^{n}, u_{i}^{n}\right)\right|,
\end{aligned}
$$


and similarly

$$
\begin{aligned}
& \sum_{i=1}^{k_{n}}\left|f\left(t_{i-1}^{n}, u_{i}^{n}\right)-f\left(t_{i-1}^{n}, u_{i-1}^{n}\right)\right| \\
& \quad \leq \sum_{i=1}^{k_{n}}\left|f\left(t_{i-1}^{n}, u_{i}^{n}\right)-f\left(t_{i}^{n}, u_{i}^{n}\right)\right|+\sum_{i=1}^{k_{n}}\left|f\left(t_{i}^{n}, u_{i}^{n}\right)-f\left(t_{i-1}^{n}, u_{i-1}^{n}\right)\right| \\
& \quad \leq \sum_{i=1}^{k_{n}}\left|f\left(t_{i}^{n}, u_{i}^{n}\right)-f\left(t_{i-1}^{n}, u_{i}^{n}\right)\right|+\sup _{n \in \mathbb{N}} \bigvee_{0}^{1} F\left(\xi^{n}\right) .
\end{aligned}
$$

Therefore, both sums in (3.3) diverge to $+\infty$ as $n \rightarrow+\infty$, if at least one of them diverges. So, we have that

$$
\lim _{n \rightarrow \infty} \sum_{i=1}^{k_{n}}\left|f\left(t_{i-1}^{n}, u_{i}^{n}\right)-f\left(t_{i-1}^{n}, u_{i-1}^{n}\right)\right|=+\infty .
$$

Fix $n \in \mathbb{N}$. To each $t_{i}^{n}$ arbitrarily assign a point $\tau_{i+1}^{n} \in\left(t_{i}^{n}, t_{i+1}^{n}\right), i=$ $0, \ldots, k_{n}-1$. The finite sequence $s_{i}^{n}$, where $i=0, \ldots, 2 k_{n}$, defined by $s_{0}^{n}:=t_{0}^{n}, \quad s_{1}^{n}:=\tau_{1}^{n}, \quad s_{2}^{n}:=t_{1}^{n}, \quad s_{3}^{n}:=\tau_{2}^{n}, \quad s_{4}^{n}:=t_{2}^{n}, \ldots, s_{2 k_{n}-1}^{n}:=\tau_{k_{n}}^{n}, \quad s_{2 k_{n}}^{n}:=t_{k_{n}}^{n}$ is a partition of $[0,1]$. Let us also define two functions:

$$
x^{n}(t)= \begin{cases}u_{i}^{n} & \text { if } t \in\left[s_{2 i-2}^{n}, s_{2 i-1}^{n}\right] \\ \frac{s_{2 i}^{n}-t}{s_{2 i}^{n}-s_{2 i-1}^{n}} u_{i}^{n}+\frac{t-s_{2 i-1}^{n}}{s_{2 i}^{n}-s_{2 i-1}^{n}} u_{i+1}^{n} & \text { for some } i \in\left\{1, \ldots, k_{n}\right\}, \\ & \text { if } t \in\left[s_{2 i-1}^{n}, s_{2 i}^{n}\right] \\ u_{k_{n}}^{n} & \text { for some } i \in\left\{1, \ldots, k_{n}-1\right\}, \\ \text { if } t \geq s_{2 k_{n}-1}^{n},\end{cases}
$$

and

$$
y^{n}(t)= \begin{cases}\frac{s_{2 i-1}^{n}-t}{s_{2 i-1}^{n}-s_{2 i-2}^{n}} u_{i-1}^{n}+\frac{t-s_{2 i-2}^{n}}{s_{2 i-1}^{n}-s_{2 i-2}^{n}} u_{i}^{n} & \text { if } t \in\left[s_{2 i-2}^{n}, s_{2 i-1}^{n}\right] \\ & \text { for some } i \in\left\{1, \ldots, k_{n}\right\}, \\ u_{i}^{n} & \text { if } t \in\left[s_{2 i-1}^{n}, s_{2 i}^{n}\right] \\ & \text { for some } i \in\left\{1, \ldots, k_{n}\right\} .\end{cases}
$$

The following table explains how the functions $x^{n}$ and $y^{n}$ are constructed (for the sake of simplicity, the upper indices $n$ at $t, \tau, s$ and $u$ are omitted):

\begin{tabular}{c|c|c|c|c|c|c|c|c|c|c|c|c} 
values of $t$ & $s_{0}$ & $s_{1}$ & $s_{2}$ & $s_{3}$ & $s_{4}$ & $s_{5}$ & $s_{6}$ & $\ldots$ & $s_{2 k_{n}-3}$ & $s_{2 k_{n}-2}$ & $s_{2 k_{n}-1}$ & $s_{2 k_{n}}$ \\
\hline 'old values' of $t$ & $t_{0}$ & $\tau_{1}$ & $t_{1}$ & $\tau_{2}$ & $t_{2}$ & $\tau_{3}$ & $t_{3}$ & $\ldots$ & $\tau_{k_{n}-1}$ & $t_{k_{n}-1}$ & $\tau_{k_{n}}$ & $t_{k_{n}}$ \\
values of $x^{n}(t)$ & $u_{1}$ & $u_{1}$ & $u_{2}$ & $u_{2}$ & $u_{3}$ & $u_{3}$ & $u_{4}$ & $\ldots$ & $u_{k_{n}-1}$ & $u_{k_{n}}$ & $u_{k_{n}}$ & $u_{k_{n}}$ \\
values of $y^{n}(t)$ & $u_{0}$ & $u_{1}$ & $u_{1}$ & $u_{2}$ & $u_{2}$ & $u_{3}$ & $u_{3}$ & $\ldots$ & $u_{k_{n}-1}$ & $u_{k_{n}-1}$ & $u_{k_{n}}$ & $u_{k_{n}}$
\end{tabular}

and the functions $x^{n}$ and $y^{n}$ are linear on each interval $\left[s_{i}, s_{i+1}\right]$. 
Observe that $x^{n}(0)=u_{1}^{n}, y^{n}(0)=u_{0}^{n}$, and furthermore

$$
\bigvee_{0}^{1} x^{n}=\sum_{i=1}^{2 k_{n}}\left|x^{n}\left(s_{i}^{n}\right)-x^{n}\left(s_{i-1}^{n}\right)\right|=\sum_{i=2}^{k_{n}}\left|u_{i}^{n}-u_{i-1}^{n}\right| \leq \bigvee_{0}^{1} \xi^{n} \leq r
$$

as well as

$$
\bigvee_{0}^{1} y^{n}=\sum_{i=1}^{2 k_{n}}\left|y^{n}\left(s_{i}^{n}\right)-y^{n}\left(s_{i-1}^{n}\right)\right|=\sum_{i=1}^{k_{n}}\left|u_{i}^{n}-u_{i-1}^{n}\right| \leq \bigvee_{0}^{1} \xi^{n} \leq r .
$$

Therefore, $\left\|x^{n}\right\|_{\mathrm{BV}} \leq 2 r$ and $\left\|y^{n}\right\|_{\mathrm{BV}} \leq 2 r$ for every $n \in \mathbb{N}$. Hence, in view of the local boundedness of the superposition operator $F$, we infer that $\left\|F\left(z^{n}\right)\right\|_{\mathrm{BV}} \leq R$, where $z^{n} \in\left\{x^{n}, y^{n}\right\}$. However, on the other hand, for every $n \in \mathbb{N}$ we have

$$
\begin{aligned}
& \sum_{i=1}^{k_{n}}\left|f\left(t_{i-1}^{n}, u_{i}^{n}\right)-f\left(t_{i-1}^{n}, u_{i-1}^{n}\right)\right| \\
& \leq \sum_{i=1}^{k_{n}}\left|f\left(t_{i-1}^{n}, u_{i}^{n}\right)-f\left(\tau_{i}^{n}, u_{i}^{n}\right)\right|+\sum_{i=1}^{k_{n}}\left|f\left(\tau_{i}^{n}, u_{i}^{n}\right)-f\left(t_{i-1}^{n}, u_{i-1}^{n}\right)\right| \\
& =\sum_{i=1}^{k_{n}}\left|f\left(t_{i-1}^{n}, x^{n}\left(t_{i-1}^{n}\right)\right)-f\left(\tau_{i}^{n}, x^{n}\left(\tau_{i}^{n}\right)\right)\right| \\
& \quad+\sum_{i=1}^{k_{n}}\left|f\left(\tau_{i}^{n}, y^{n}\left(\tau_{i}^{n}\right)\right)-f\left(t_{i-1}^{n}, y^{n}\left(t_{i-1}^{n}\right)\right)\right| \\
& =\sum_{i=1}^{k_{n}}\left|f\left(s_{2 i-1}^{n}, x^{n}\left(s_{2 i-1}^{n}\right)\right)-f\left(s_{2 i-2}^{n}, x^{n}\left(s_{2 i-2}^{n}\right)\right)\right| \\
& \quad+\sum_{i=1}^{k_{n}}\left|f\left(s_{2 i-1}^{n}, y^{n}\left(s_{2 i-1}^{n}\right)\right)-f\left(s_{2 i-2}^{n}, y^{n}\left(s_{2 i-2}^{n}\right)\right)\right| \\
& \leq \bigvee_{0}^{1} F\left(x^{n}\right)+\bigvee_{0}^{1} F\left(y^{n}\right) \leq 2 R,
\end{aligned}
$$

which contradicts (3.4). This shows that the implication $(\mathrm{a}) \Rightarrow(\mathrm{b})$ holds.

REMARK 3.9. Let us add that Theorem 3.1 and Proposition 3.3 can be obtained as corollaries to Theorem 3.8.

\section{The case of locally bounded functions}

In this section, unless stated otherwise, we assume that $f:[0,1] \times \mathbb{R} \rightarrow \mathbb{R}$ maps bounded sets into bounded sets and that $F$ is the nonautonomous superposition operator generated by $f$. For brevity, let $l_{\alpha}(t):=\max \{0, t-\alpha\}$ and $r_{\alpha}(t):=\min \{1, t+\alpha\}$ for $t \in[0,1]$ and $\alpha \in(0,+\infty)$.

Reasoning similar to the proof of [24, Lemma 1] leads to the following 
Lemma 4.1. Let $x:[0,1] \rightarrow \mathbb{R}$. Then $F(x) \notin \mathrm{BV}[0,1]$ if and only if there exists $t \in[0,1]$ such that

$$
\bigvee_{l_{\alpha}(t)}^{r_{\alpha}(t)} F(x)=+\infty \quad \text { for every } \alpha>0
$$

ProOF. Let us note that it suffices to prove the implication ' $\Rightarrow$ ', and therefore suppose that (4.1) does not hold. Then, for any $t \in[0,1]$ there exists $\alpha_{t}>0$ such that

$$
\bigvee_{l_{\alpha_{t}}(t)}^{r_{\alpha_{t}}(t)} F(x)=: M_{t}<+\infty
$$

The family of sets $\left\{\left(t-\alpha_{t}, t+\alpha_{t}\right): t \in[0,1]\right\}$ is an open cover of $[0,1]$, which implies that for some points $0 \leq t_{0}<t_{1}<\ldots<t_{k} \leq 1$ we have $[0,1] \subset$ $\left(t_{0}-\alpha_{t_{0}}, t_{0}+\alpha_{t_{0}}\right) \cup \ldots \cup\left(t_{k}-\alpha_{t_{k}}, t_{k}+\alpha_{t_{k}}\right)$ and

$$
\bigvee_{0}^{1} F(x) \leq \sum_{i=0}^{k} \bigvee_{l_{\alpha_{t_{i}}}\left(t_{i}\right)}^{r_{\alpha_{t_{i}}}\left(t_{i}\right)} F(x) \leq k \max \left\{M_{t_{0}}, \ldots, M_{t_{k}}\right\}<+\infty
$$

Now, we are going to apply Lemma 4.1 to the following technical result, which is crucial for our further considerations.

Lemma 4.2. Suppose that there exists $x \in B_{\mathrm{BV}}(0, r)$, where $r>0$, such that $F(x) \notin \mathrm{BV}[0,1]$ and let $t \in[0,1]$ satisfy condition (4.1). Then for every $\delta>0$ there exists $u \in[-r, r]$ such that for every $q \in \mathbb{N}$ there exist positive integers $c_{q}, d_{q}$ and a finite collection of points $l_{1 / c_{q}}(t) \leq t_{0}^{q}<t_{1}^{q}<\ldots<t_{d_{q}}^{q} \leq r_{1 / c_{q}}(t)$ such that the following properties hold: $x\left(t_{i}^{q}\right) \in[u-\delta, u+\delta]$ for $i=0, \ldots, d_{q}$, $c_{q} \rightarrow+\infty$ as $q \rightarrow+\infty$ and

$$
\lim _{q \rightarrow \infty} \sum_{i=1}^{d_{q}}\left|f\left(t_{i}^{q}, x\left(t_{i}^{q}\right)\right)-f\left(t_{i-1}^{q}, x\left(t_{i-1}^{q}\right)\right)\right|=+\infty .
$$

Proof. Fix $\delta>0$ (without loss of generality we may assume that $6 r / \delta$ is a positive integer greater than or equal to 2$)$. Since $t \in[0,1]$ satisfies (4.1), for each $n \in \mathbb{N}$ we can choose a number $k_{n} \in \mathbb{N}$ and a finite partition $l_{1 / n}(t)=\tau_{0}^{n}<$ $\ldots<\tau_{k_{n}}^{n}=r_{1 / n}(t)$ of the interval $\left[l_{1 / n}(t), r_{1 / n}(t)\right]$ such that

$$
\sum_{i=1}^{k_{n}}\left|f\left(\tau_{i}^{n}, x\left(\tau_{i}^{n}\right)\right)-f\left(\tau_{i-1}^{n}, x\left(\tau_{i-1}^{n}\right)\right)\right| \geq n .
$$

The function $f$ is locally bounded, and therefore

$$
M_{r}:=\sup \{|f(\tau, w)|: \tau \in[0,1] \text { and } w \in[-r, r]\}<+\infty \text {, }
$$

which, in particular, shows that $k_{n} \rightarrow+\infty$ as $n \rightarrow+\infty$. 
Now, let us define

$$
u^{0}:=-r \quad \text { and } \quad u^{j+1}:=u^{j}+\frac{\delta}{3} \quad \text { for } j=0, \ldots, s-1,
$$

where $s=6 r / \delta$. Then, the intervals $\left[u^{j}-\delta / 6, u^{j}+\delta / 6\right]$, where $j=0, \ldots, s$, cover the interval $[-r, r]$. Moreover,

$\left[u^{j}-\delta / 6, u^{j}+\delta / 6\right] \cap\left[u^{j+1}-\delta / 6, u^{j+1}+\delta / 6\right]=\left\{u^{j}+\delta / 6\right\} \quad$ for $j=0, \ldots, s-1$, and we have

$$
|u-w| \geq \delta / 3 \quad \text { for } u \in\left[u^{i}-\delta / 6, u^{i}+\delta / 6\right], w \in\left[u^{j}-\delta / 6, u^{j}+\delta / 6\right]
$$
and $i, j$ with $|i-j|>1$.

For every $n \in \mathbb{N}$ we have

$$
\left\{x\left(\tau_{i}^{n}\right): i=0,1, \ldots, k_{n}\right\} \subset \bigcup_{j=0}^{s}\left[u^{j}-\delta / 6, u^{j}+\delta / 6\right],
$$

and then to each $i \in\left\{0, \ldots, k_{n}\right\}$ we can assign a number $j^{n}(i) \in\{0, \ldots, s\}$ by

$$
j^{n}(i):=\min \left\{j: x\left(\tau_{i}^{n}\right) \in\left[u^{j}-\delta / 6, u^{j}+\delta / 6\right]\right\} .
$$

For every $n \in \mathbb{N}$ either

(a) $\left|j^{n}(i)-j^{n}(0)\right|<2$ for all $i \in\left\{0, \ldots, k_{n}\right\}$, which means that $x\left(\tau_{i}^{n}\right) \in$ $\left[u^{j^{n}(0)}-\delta / 2, u^{j^{n}(0)}+\delta / 2\right]$ for all $i \in\left\{0, \ldots, k_{n}\right\}$, or

(b) there exists a $\delta$-jump, i.e. there exists the first index $i^{*} \in\left\{1, \ldots, k_{n}\right\}$ such that $\left|j^{n}\left(i^{*}\right)-j^{n}(0)\right| \geq 2$, and then $\left|x\left(\tau_{i^{*}}^{n}\right)-x\left(\tau_{0}^{n}\right)\right|>\delta / 3$.

Taking $i^{*}$ in place of 0 and repeating the above reasoning, we get that either there is a $\delta$-jump for some $i^{* *}>i^{*}$ or not, and so on. Let us note that since $\|x\|_{\mathrm{BV}} \leq r$, the number of the consecutive $\delta$-jumps 'at the level' $n$, which will be denoted by $m_{n}$, is at most $3 r / \delta$ (observe that the upper bound for the number of $\delta$-jumps does not depend on $n$ ). Indeed, if the function $x$ has consecutive $\delta$-jumps between the points $\left(^{3}\right) \tau_{0}^{n}, \ldots, \tau_{m_{n}}^{n}$, then

$$
r \geq \sum_{i=0}^{m_{n}-1}\left|x\left(\tau_{i}^{n}\right)-x\left(\tau_{i+1}^{n}\right)\right| \geq \frac{1}{3} \delta m_{n} .
$$

This procedure leads to the following definition of the sets $I_{l}^{n}$, for $l=0, \ldots, m_{n}$ :

$$
I_{l}^{n}:= \begin{cases}\left\{i \in \mathbb{N}: i_{l}^{n}+1 \leq i \leq i_{l+1}^{n}-1\right\} & \text { if } i_{l+1}^{n}<k_{n}+1, \\ \left\{i \in \mathbb{N}: i_{l}^{n}+1 \leq i \leq k_{n}\right\} & \text { if } i_{l+1}^{n}=k_{n}+1,\end{cases}
$$

where $i_{0}^{n}:=0$ and $i_{l+1}^{n}:=\min \left(\left\{i \in\left\{i_{l}^{n}, \ldots, k_{n}\right\}:\left|j^{n}\left(i_{l}^{n}\right)-j^{n}(i)\right| \geq 2\right\} \cup\left\{k_{n}+1\right\}\right)$.

$\left({ }^{3}\right)$ For the sake of simplicity, we assume here that the consecutive $\delta$-jumps at the level $n$ appear between the first $m_{n}+1$ points of the partition. 
Let us note that $\left(^{4}\right)$

$$
\begin{aligned}
\sum_{i=1}^{k_{n}}\left|f\left(\tau_{i}^{n}, x\left(\tau_{i}^{n}\right)\right)-f\left(\tau_{i-1}^{n}, x\left(\tau_{i-1}^{n}\right)\right)\right|= & \sum_{l=0}^{m_{n}} \sum_{i \in I_{l}^{n}}\left|f\left(\tau_{i}^{n}, x\left(\tau_{i}^{n}\right)\right)-f\left(\tau_{i-1}^{n}, x\left(\tau_{i-1}^{n}\right)\right)\right| \\
& +\sum_{l=1}^{m_{n}}\left|f\left(\tau_{i_{l}^{n}}^{n}, x\left(\tau_{i_{l}^{n}}^{n}\right)\right)-f\left(\tau_{i_{l}^{n}-1}^{n}, x\left(\tau_{i_{l}^{n}-1}^{n}\right)\right)\right|,
\end{aligned}
$$

and since

$$
\sum_{l=1}^{m_{n}}\left|f\left(\tau_{i_{l}^{n}}^{n}, x\left(\tau_{i_{l}^{n}}^{n}\right)\right)-f\left(\tau_{i_{l}^{n}-1}^{n}, x\left(\tau_{i_{l}^{n}-1}^{n}\right)\right)\right| \leq 2 m_{n} M_{r} \leq s M_{r},
$$

we infer that for $n$ sufficiently large there exists $l_{n} \in\left\{0, \ldots, m_{n}\right\}$ such that

$$
\sum_{i \in I_{l_{n}}^{n}}\left|f\left(\tau_{i}^{n}, x\left(\tau_{i}^{n}\right)\right)-f\left(\tau_{i-1}^{n}, x\left(\tau_{i-1}^{n}\right)\right)\right| \geq \frac{\delta}{\delta+3 r}\left(n-s M_{r}\right) ;
$$

in particular, $I_{l_{n}}^{n}$ is non-empty. Since $m_{n} \leq 3 r / \delta$ for every $n \in \mathbb{N}$, there exists a strictly increasing sequence $\left(n_{q}\right)_{q \in \mathbb{N}}$ of positive integers divergent to $+\infty$ and a number $l \in\left\{0, \ldots, \max _{n \in \mathbb{N}} m_{n}\right\}$ independent of $n$ for which

$$
\lim _{q \rightarrow \infty} \sum_{i \in I_{l}^{n_{q}}}\left|f\left(\tau_{i}^{n_{q}}, x\left(\tau_{i}^{n_{q}}\right)\right)-f\left(\tau_{i-1}^{n_{q}}, x\left(\tau_{i-1}^{n_{q}}\right)\right)\right|=+\infty .
$$

Similarly, there is $j \in\{0, \ldots, s\}$ for which $x\left(\tau_{i_{l}^{n_{q}}}^{n_{q}}\right) \in\left[u^{j}-\delta / 6, u^{j}+\delta / 6\right]$ for infinitely many $q \in \mathbb{N}$. Therefore, passing to a subsequence if necessary, we may assume that $x\left(\tau_{i_{l}^{n_{q}}}^{n_{q}}\right) \in\left[u^{j}-\delta / 6, u^{j}+\delta / 6\right]$ for every $q \in \mathbb{N}$. So, by the definition of the set $I_{l}^{n_{q}}$ we obtain that $\left|u^{j}-x\left(\tau_{i}^{n_{q}}\right)\right| \leq \delta$ for $i \in I_{l}^{n_{q}}$ and $q \in \mathbb{N}$.

Finally, set

$$
u:=u^{j}, \quad c_{q}:=n_{q}, \quad d_{q}:=\left|I_{l}^{n_{q}}\right|, \quad t_{h}^{q}:=\tau_{i_{l}^{n_{q}}+h}^{n_{q}} \quad \text { for } h=0, \ldots, d_{q} ;
$$

here $|A|$ denotes the cardinality of the set $A$. Together with condition (4.5), this proves our assertion.

LEMma 4.3. Let the function $x \in B_{\mathrm{BV}}(0, r)$, where $r>0$, be such that $F(x) \notin \mathrm{BV}[0,1]$ and let $t \in[0,1]$ satisfy condition (4.1). Then, there exists $u \in[-r, r]$ such that for every $\varepsilon>0$ and every sequence $\left(\delta_{n}\right)_{n \in \mathbb{N}}$ of positive numbers convergent to 0 there exist sequences of positive integers $k_{n}:=k\left(\delta_{n}, \varepsilon\right)$ and finite collections of points $l_{\varepsilon}(t) \leq t_{0}^{\delta_{n}, \varepsilon}<t_{1}^{\delta_{n}, \varepsilon}<\ldots<t_{k_{n}}^{\delta_{n}, \varepsilon} \leq r_{\varepsilon}(t)$ for which the following conditions hold: $x\left(t_{i}^{\delta_{n}, \varepsilon}\right) \in\left[u-\delta_{n}, u+\delta_{n}\right]$, for $i=1, \ldots, k_{n}$, and

$$
\lim _{n \rightarrow \infty} \sum_{i=1}^{k_{n}}\left|f\left(t_{i}^{\delta_{n}, \varepsilon}, x\left(t_{i}^{\delta_{n}, \varepsilon}\right)\right)-f\left(t_{i-1}^{\delta_{n}, \varepsilon}, x\left(t_{i-1}^{\delta_{n}, \varepsilon}\right)\right)\right|=+\infty .
$$

$\left(^{4}\right)$ If $I_{l}^{n}=\emptyset$, then, by definition, the sum corresponding to the set $I_{l}^{n}$ equals zero. 
ProOF. Fix an arbitrary sequence $\left(\gamma_{n}\right)_{n \in \mathbb{N}}$ of positive numbers convergent to 0 and suppose $u_{n} \in[-r, r]$ corresponds to $\gamma_{n}$ according to Lemma 4.2 (with $\left.\delta=\gamma_{n}\right)$. If we denote an accumulation point of the sequence $\left(u_{n}\right)_{n \in \mathbb{N}}$ by $u$, then it is not difficult to show that the point $u$ satisfies the assertion.

Is is clear that if for a function $x \in B_{\mathrm{BV}}(0, r)$, where $r>0$, there exist points $t \in[0,1]$ and $u \in[-r, r]$ such that the claim of Lemma 4.3 is satisfied, then $F(x) \notin \mathrm{BV}[0,1]$. Therefore, we can state

Theorem 4.4. Let $x \in B_{\mathrm{BV}}(0, r)$ for some $r>0$. Then $F(x) \in \mathrm{BV}[0,1]$ if and only if for any $t \in[0,1]$ and $u \in[-r, r]$ there exist $\varepsilon>0, \delta>0$ and $M>0$ such that $l_{\varepsilon}(t) \leq t_{0}<t_{1}<\ldots<t_{k} \leq r_{\varepsilon}(t)$ and $x\left(t_{i}\right) \in[u-\delta, u+\delta]$ for $i \in\{0, \ldots, k\}, k \in \mathbb{N}$, imply

$$
\sum_{i=1}^{k}\left|f\left(t_{i}, x\left(t_{i}\right)\right)-f\left(t_{i-1}, x\left(t_{i-1}\right)\right)\right|<M .
$$

Before we restate Theorem 4.4 we need the following

Definition 4.5. (a) Let $A \subset \mathbb{R}$ be a non-empty set and let $a, b \in \mathbb{R}$ be such that $a<b$. A finite sequence $\left(t_{i}, u_{i}\right)_{i=0}^{k}$ is called a flagged partition of $[a, b] \times A$, if $a \leq t_{0}<t_{1}<\ldots<t_{k} \leq b$ and $u_{i} \in A$ for $i=0, \ldots, k$.

(b) If $V^{1}$ and $V^{2}$ are flagged partitions of $[a, b] \times A$, then $V^{2}$ is called a condensation of $V^{1}$ (which we will denote by $V^{1} \preceq V^{2}$ ), if $V^{1}$ is a subsequence of $V^{2}$.

(c) A sequence $\left(V^{n}\right)_{n \in \mathbb{N}}$ of flagged partitions of $[a, b] \times A$ is called a condensation sequence of $[a, b] \times A$, if $V^{n} \preceq V^{n+1}$ for $n \in \mathbb{N}$.

(d) A condensation sequence $\left(V^{n}\right)_{n \in \mathbb{N}}$ of $[a, b] \times A$, where $V^{n}=\left(t_{i}^{n}, u_{i}^{n}\right)_{i=0}^{k_{n}}$, is called proper, if $\sup _{n} \sum_{i=1}^{k_{n}}\left|u_{i}^{n}-u_{i-1}^{n}\right|<+\infty$.

THEOREM 4.6. The operator $F$ maps BV[0,1] into itself if and only if for any $t \in[0,1]$ and $u \in \mathbb{R}$ there exist $\varepsilon>0$ and $\delta>0$ such that for any proper condensation sequence $\left(V^{n}\right)_{n \in \mathbb{N}}$ of $\left[l_{\varepsilon}(t), r_{\varepsilon}(t)\right] \times[u-\delta, u+\delta]$, where $V^{n}=\left(t_{i}^{n}, u_{i}^{n}\right)_{i=0}^{k_{n}}$, it holds

$$
\sup _{n} \sum_{i=1}^{k_{n}}\left|f\left(t_{i}^{n}, u_{i}^{n}\right)-f\left(t_{i-1}^{n}, u_{i-1}^{n}\right)\right|<+\infty .
$$

Proof. Suppose that there exists $x \in \mathrm{BV}[0,1]$ such that $F(x) \notin \mathrm{BV}[0,1]$. Then, the existence of a desired proper condensation sequence $\left(V^{n}\right)_{n \in \mathbb{N}}$ of $\left[l_{\varepsilon}(t), r_{\varepsilon}(t)\right] \times[u-\delta, u+\delta]$ follows directly from Theorem 4.4 (one has to apply it denumerably many times with $M=n$ ).

Suppose now that the condition stated in Theorem 4.6 does not hold. Then, there exist $t \in[0,1]$ and $u \in \mathbb{R}$ together with a proper condensation sequence 
$\left(V^{n}\right)_{n \in \mathbb{N}}$ of $[0,1] \times[u-1, u+1]$ of the form $V^{n}=\left(t_{i}^{n}, u_{i}^{n}\right)_{i=0}^{k_{n}}$ such that

$$
\sup _{n} \sum_{i=1}^{k_{n}}\left|f\left(t_{i}^{n}, u_{i}^{n}\right)-f\left(t_{i-1}^{n}, u_{i-1}^{n}\right)\right|=+\infty
$$

(here we put $\varepsilon=\delta=1$ ).

For a fixed $n \in \mathbb{N}$ let $x_{n}:[0,1] \rightarrow \mathbb{R}$ be a function which is linear on each interval $\left[t_{i-1}^{n}, t_{i}^{n}\right]$ for $i=1, \ldots, k_{n}$, and whose graph is spanned by the points $\left(t_{i}^{n}, u_{i}^{n}\right), i=0, \ldots, k$, and is parallel to the $x$-axis on the intervals $\left[0, t_{0}^{n}\right]$ and $\left[t_{k_{n}}^{n}, 1\right]$. Since, the sequence $\left(V^{n}\right)_{n \in \mathbb{N}}$ is proper, we infer that

$$
\sup _{n \in \mathbb{N}} \bigvee_{0}^{1} x_{n}=\sup _{n \in \mathbb{N}} \sum_{i=1}^{k_{n}}\left|u_{i}^{n}-u_{i-1}^{n}\right|<+\infty .
$$

Thus, the sequence $\left(x_{n}\right)_{n \in \mathbb{N}}$ is bounded in $\mathrm{BV}[0,1]$, and therefore, in view of Helly's extraction theorem, there is a subsequence $\left(x_{n_{k}}\right)_{k \in \mathbb{N}}$ and a function $x \in \mathrm{BV}[0,1]$ such that $x(t)=\lim _{k \rightarrow \infty} x_{n_{k}}(t)$ for every $t \in[0,1]$. Let us note that, due to the fact that $\left(V^{n}\right)_{n \in \mathbb{N}}$ is a condensation sequence, $x\left(t_{i}^{n}\right)=u_{i}^{n}$ for every $i \in\left\{0, \ldots, k_{n}\right\}$ and $n \in \mathbb{N}$. This, in connection with (4.7), shows that $F(x) \notin \mathrm{BV}[0,1]$.

\section{Nonautonomous superposition operators - a separable variables case}

In this section we are going to study nonlinear superposition operators which are generated by functions with separable variables, that is, functions of the form $(t, u) \mapsto f(t) g(u)$, where $f:[0,1] \rightarrow \mathbb{R}$ and $g: \mathbb{R} \rightarrow \mathbb{R}$. Although the results of this section may be treated as corollaries to Theorem 3.8, we are going to present them with proofs, which (due to the form of the considered generators) are based on different techniques, and therefore can be significantly simplified.

The following simple result explains when a nonautonomous superposition operator acting in a function space is generated by a function with separable variables.

TheOREM 5.1. Let $X$ be a vector space over $\mathbb{R}$ satisfying the following conditions:

(a) $X$ is a vector subspace of the vector space all real-valued functions defined on $[0,1]$ considered with the standard pointwise addition and multiplication by scalars;

(b) $X$ contains all constant functions.

Moreover, assume that $F$ is a nonautonomous superposition operator which maps the vector space $X$ into itself. The superposition operator $F$ is generated by a function of the form $(t, u) \mapsto f(t) g(u)$, where $f:[0,1] \rightarrow \mathbb{R}$ and $g: \mathbb{R} \rightarrow \mathbb{R}$ if and only if there exists $u_{0} \in \mathbb{R}$ such that for every $u \in \mathbb{R}$ there is $a_{u} \in \mathbb{R}$ such 
that $F\left(x_{u}\right)=a_{u} F\left(x_{u_{0}}\right)$. Furthermore, if $F \not \equiv 0$, then the functions $f$ and $g$ are uniquely determined up to constant factors $\alpha$ and $\beta$, respectively, such that $\alpha \beta=1$.

Proof. Clearly, if the operator $F$ is generated by a function with separable variables, then either $g \equiv 0$ and $u_{0}:=1$ and $a_{u}:=0$ for every $u \in \mathbb{R}$, or $g\left(u_{0}\right) \neq 0$ for some $u_{0} \in \mathbb{R}$, and then $a_{u}:=g(u) / g\left(u_{0}\right)$.

Now, we will prove the necessity part, that is, that the generator of the operator $F$, say $h:[0,1] \times \mathbb{R} \rightarrow \mathbb{R}$, can be represented in the form $h(t, u)=$ $f(t) g(u)$ for some functions $f:[0,1] \rightarrow \mathbb{R}$ and $g: \mathbb{R} \rightarrow \mathbb{R}$. From the assumptions it follows that we have

$$
h(t, u)=a_{u} h\left(t, u_{0}\right) \quad \text { for every } t \in[0,1] \text { and } u \in \mathbb{R} .
$$

If $h\left(t, u_{0}\right)=0$ for any $t \in[0,1]$, then $h \equiv 0$, and it suffices to take $f \equiv 0$ and $g \equiv 0$. So we may assume that $h\left(t_{0}, u_{0}\right) \neq 0$ for some $t_{0} \in[0,1]$. Define functions $f$ and $g$ by the following formulas:

$$
f(t)=h\left(t, u_{0}\right) \quad \text { and } \quad g(u)=\frac{h\left(t_{0}, u\right)}{h\left(t_{0}, u_{0}\right)} .
$$

Then $h(t, u)=f(t) g(u)$ for $t \in[0,1]$ and $u \in \mathbb{R}$.

Finally, suppose that the functions $f_{1}:[0,1] \rightarrow \mathbb{R}$ and $g_{1}: \mathbb{R} \rightarrow \mathbb{R}$ are such that

$$
f(t) g(u)=f_{1}(t) g_{1}(u) \quad \text { for all }(t, u) \in[0,1] \times \mathbb{R} .
$$

Since $F \not \equiv 0$, there exists a point $(s, w) \in[0,1] \times \mathbb{R}$ such that $f(s) g(w) \neq 0$. Then, putting

$$
\alpha:=\frac{g_{1}(w)}{g(w)} \quad \text { and } \quad \beta:=\frac{f_{1}(s)}{f(s)},
$$

we have $\alpha \beta=1$ as well as

$$
f(t)=\alpha f_{1}(t) \quad \text { and } \quad g(u)=\beta g_{1}(u) \quad \text { for } t \in[0,1] \text { and } u \in \mathbb{R} .
$$

This ends the proof.

Clearly, if $f \equiv 0$, then from the fact that the superposition operator $F$, generated by the function $(t, u) \mapsto f(t) g(u)$, maps the space $X$ into itself, nothing can be inferred about properties of $g$. However, the more is known about the behaviour of the function $f$ at points of its continuity, the more can be proved about $g$.

Now, let us pass on to the space BV $[0,1]$.

TheOrem 5.2. Let $f:[0,1] \rightarrow \mathbb{R}$ and $g: \mathbb{R} \rightarrow \mathbb{R}$ be two functions satisfying the following conditions:

(a) there exists $u_{0} \in \mathbb{R}$ such that $g\left(u_{0}\right) \neq 0$;

(b) there exists a point $t_{0} \in[0,1]$ of continuity of $f$ such that $f\left(t_{0}\right) \neq 0$. 
Then the nonautonomous superposition operator $F$, generated by the function $(t, u) \mapsto f(t) g(u)$, maps the space $\mathrm{BV}[0,1]$ into itself if and only if:

(c) $f \in \mathrm{BV}[0,1]$;

(d) g satisfies a local Lipschitz condition.

Proof. The sufficiency of conditions (c) and (d) is obvious, since BV $[0,1]$ is an algebra (cf. also Theorem 2.2).

Now, we will prove the necessity part. In view of the assumptions, $F\left(x_{u_{0}}\right) \in$ $\mathrm{BV}[0,1]$, which implies that $f \in \mathrm{BV}[0,1]$. Indeed

$$
f(t)=\frac{1}{g\left(u_{0}\right)} f(t) g\left(u_{0}\right)=\frac{1}{g\left(u_{0}\right)} F\left(x_{u_{0}}\right)(t) \quad \text { for } t \in[0,1],
$$

which proves our claim.

Furthermore, by assumption (b) there exist an interval $[c, d] \subset[0,1]$ with a non-empty interior and a constant $M>0$ such that $|f(t)| \geq M$ for all $t \in[c, d]$. Now, let $\xi:[c, d] \rightarrow \mathbb{R}$ be an arbitrary function of bounded variation and let $x:[0,1] \rightarrow \mathbb{R}$ be its BV-extension, that is, we set

$$
x(t)= \begin{cases}\xi(c) & \text { if } t \in[0, c], \\ \xi(t) & \text { if } t \in[c, d], \\ \xi(d) & \text { if } t \in[d, 1] .\end{cases}
$$

Then $F(x) \in \mathrm{BV}[0,1]$ and

$$
g(\xi(t))=\frac{1}{f(t)} \cdot f(t) g(\xi(t))=\frac{1}{f(t)} \cdot F(x)(t) \quad \text { for } t \in[c, d],
$$

which, in view of $[16$, Theorem 6.1.11, p. 120], proves that the function $g$ generates an autonomous superposition operator that maps the space $\mathrm{BV}[c, d]$ into itself. Therefore, by Theorem 2.2, the function $g$ satisfies a local Lipschitz condition.

REMARK 5.3. Let us observe that conditions (c) and (d) of Theorem 5.2 guarantee that the superposition operator $F$ generated by the function $(t, u) \mapsto$ $f(t) g(u)$ is locally bounded. Therefore, in that case, similarly to the autonomous case, the fact that the superposition operator $F$ maps the Banach space BV $[0,1]$ into itself implies its local boundedness.

Now, let us consider the case when the function $f$ vanishes at each point of continuity.

Theorem 5.4. Let $f:[0,1] \rightarrow \mathbb{R}$ and $g: \mathbb{R} \rightarrow \mathbb{R}$ be two functions, and assume that

(a) $f(t)=0$ at every point $t \in[0,1]$ of continuity of $f$.

The nonautonomous superposition operator $F$, generated by the function $(t, u) \mapsto$ $f(t) g(u)$, maps the space $\mathrm{BV}[0,1]$ into itself and is locally bounded, whenever 
(b) $f \in \mathrm{BV}[0,1]$;

(c) $g$ is locally bounded.

Proof. First, let us observe that the set $D_{f}$ of points of discontinuity of $f$ is at most denumerable and

$$
\sum_{t \in D_{f}}|f(t)| \leq \bigvee_{0}^{1} f<+\infty .
$$

Then, for any $x \in \mathrm{BV}[0,1]$, we have

$$
F(x)(t)= \begin{cases}f(t) g(x(t)) & \text { if } t \in D_{f}, \\ 0 & \text { if } t \notin D_{f},\end{cases}
$$

and hence

$$
\bigvee_{0}^{1} F(x) \leq 2 M \sum_{t \in D_{f}}|f(t)|,
$$

where the constant $M>0$ is such that $|g(u)| \leq M$ for $u \in\left[-\|x\|_{\mathrm{BV}},\|x\|_{\mathrm{BV}}\right]$. This proves that the superposition operator maps the Banach space BV $[0,1]$ into itself. The local boundedness of the superposition operator $F$ follows immediately from the above estimate.

REMARK 5.5. If $f \in \mathrm{BV}[0,1]$, then assumption (a) of Theorem 5.4 implies that $f(t) \neq 0$ if and only if $t \in D_{f}$.

Indeed, if $f(t) \neq 0$, then clearly $t \in D_{f}$. Now, let us suppose that $f(t)=0$ and let $\left(t_{n}\right)_{n \in \mathbb{N}}$ be an arbitrary sequence in $[0,1]$ which is convergent to $t$ and such that $t_{n} \neq t$ for all $n \in \mathbb{N}$. If $t_{n} \notin D_{f}$ for every $n \in \mathbb{N}$, then clearly $f\left(t_{n}\right) \rightarrow f(t)$ as $n \rightarrow+\infty$. On the other hand, if $t_{n} \in D_{f}$ for every $n \in \mathbb{N}$, then since $f \in \mathrm{BV}[0,1]$, we have

$$
\sum_{t \in D_{f}}|f(t)| \leq \bigvee_{0}^{1} f<+\infty,
$$

and hence $\left|f\left(t_{n}\right)\right| \rightarrow 0$ as $n \rightarrow+\infty$, which shows that $f\left(t_{n}\right) \rightarrow f(t)$ and proves that $t \notin D_{f}$.

Let us observe that if we drop the assumption that $f$ is of bounded variation, then the claim that assumption (a) of Theorem 5.4 implies that $f(t) \neq 0$ if and only if $t \in D_{f}$, is no longer true. The function $h:[0,1] \rightarrow \mathbb{R}$ defined by

$$
h(t)= \begin{cases}1 & \text { if } t=1 / n \text { for } n \in \mathbb{N}, \\ 0 & \text { if } t \neq 1 / n \text { for } n \in \mathbb{N}\end{cases}
$$

is not continuous at 0 , but $h(0)=0$.

From Proposition 3.5, Theorem 3.7 and Theorem 5.4 (cf. also the proof of Theorem 5.2) we get the following 
Corollary 5.6. Let $f:[0,1] \rightarrow \mathbb{R}$ and $g: \mathbb{R} \rightarrow \mathbb{R}$ be two functions satisfying the following conditions:

(a) there exist $t_{0} \in[0,1]$ and $u_{0} \in \mathbb{R}$ such that $f\left(t_{0}\right) \neq 0$ and $g\left(u_{0}\right) \neq 0$;

(b) $f(t)=0$ at every point $t \in[0,1]$ of continuity of $f$.

Then the nonautonomous superposition operator $F$ generated by the function $(t, u) \mapsto f(t) g(u)$ maps the space $\mathrm{BV}[0,1]$ into itself and is locally bounded if and only if

(c) $f \in \mathrm{BV}[0,1]$;

(d) $g$ is locally bounded.

Now, we will show that the converse of the claim of Theorem 5.4 is true under certain additional condition concerning the cardinality of the set $D_{f}$ of points of discontinuity of $f$.

Theorem 5.7. Let $f:[0,1] \rightarrow \mathbb{R}$ and $g: \mathbb{R} \rightarrow \mathbb{R}$ be two functions satisfying the following conditions:

(a) there exists $u_{0} \in \mathbb{R}$ such that $g\left(u_{0}\right) \neq 0$;

(b) the set $D_{f}$ is denumerable and $f(t)=0$ at every point $t \in[0,1]$ of continuity of $f$.

Then the nonautonomous superposition operator $F$, generated by the function $(t, u) \mapsto f(t) g(u)$, maps the space $\mathrm{BV}[0,1]$ into itself if and only if

(c) $f \in \mathrm{BV}[0,1]$;

(d) $g$ is locally bounded.

Proof. The sufficiency of conditions (c) and (d) follows from Theorem 5.4. Therefore, we will pass onto the necessity part.

If $f \notin \mathrm{BV}[0,1]$, then obviously the function $F\left(x_{u_{0}}\right)$ is not of bounded variation, which ends the first part of the proof.

Now, in view of Proposition 3.6, for a given $r>0$ the set

$$
T_{r}=\left\{t \in[0,1]: \sup _{u \in[-r, r]}|f(t) g(u)|=+\infty\right\}
$$

is finite. Since the set $D_{f}$ is denumerable, we infer that there exists $t_{0} \in D_{f} \backslash T_{r}$, and hence $N_{r}:=\sup _{u \in[-r, r]}\left|f\left(t_{0}\right) g(u)\right|<+\infty$. Moreover, $f\left(t_{0}\right) \neq 0$ (see Remark 5.5), and thus $\sup _{u \in[-r, r]}|g(u)| \leq N_{r} /\left|f\left(t_{0}\right)\right|$, which proves the local boundedness of the function $g$.

\section{REFERENCES}

[1] L. Ambrosio, N. Fusco And D. Pallara, Functions of Bounded Variation and Free Discontinuity Problems, Oxford Mathematical Monographs, Oxford Science Publications, Clarendon Press, Oxford, 2000. 
[2] J. Appell, J. Banaś And N. Merentes, Bounded variation and around, De Gruyter Series in Nonlinear Analysis and Applications, vol. 17, De Gruyter, Berlin, 2014.

[3] J. Appell, N. Guanda, N. Merentes and J.L. Sanchez, Boundedness and continuity properties of nonlinear composition operators: a survey, Comm. Appl. Anal. 15 (2011), $153-182$.

[4] J. Appell And P.P. ZabrĕKo, Nonlinear Superposition Operators, Cambridge University Press, 1990.

[5] F. Brauer, Constant rate harvesting of populations governed by Volterra integral equations, J. Math. Anal. Appl., 56 (1976), 18-27.

[6] D. Bugajewska, On the superposition operator in the space of functions of bounded variation, revisited, Math. Comput. Modelling 52 (2010), 791-796.

[7] D. Bugajewski, On BV-solutions of some nonlinear integral equations, Integral Equations Operator Theory 46 (2003), 387-398.

[8] C. Castaing and M.D.P. Monteiro Marques, BV periodic solutions of an evolution problem associated with continuous moving convex sets, Set-Valued Anal. 3 (1995), 381399.

[9] V.G. ČElidze And A.G. DžvarŠĚš́vili, The Theory of the Denjoy Integral and Some Applications, Series in Real Analysis, vol. 3, World Scientific Publishing Co. Inc., Teaneck, NJ, 1989 (transl. from the Russian, with a preface and an appendix by P.S. Bullen).

[10] E. Coddington and N. Levinson, Theory of Ordinary Differential Equations, McGrawHill Book Company, New York-Toronto-London, International Series in Pure and Applied Mathematics, 1955.

[11] H. Federer, Geometric Measure Theory, Springer, Berlin, Heidelberg, New York, 1969.

[12] H. HARris And D. LAIBSOn, Dynamic choices of hyperbolic consumers, Econometrica 69 (2001), 935-957.

[13] S.I. Hudjaev And A.I. Volpert, Analysis in Classes of Discontinuous Functions and Equations of Mathematical Physics, Mechanics: Analysis, vol. 8, Martinus Nijhoff Publishers, Dordrecht, 1985.

[14] C. Jordan, Sur la série de Fourier, C.R. Acad. Sci. Paris 2 (1881), 228-230.

[15] M. Josephy, Composing functions of bounded variation, Proc. Amer. Math. Soc. 83 (1981), 354-356.

[16] R. Kannan and C.K. Krueger, Advanced Analysis on the Real Line, Universitext, Springer, New York, 1996.

[17] P. KASPRZAK AND P. MAĆKOWIAK, Local boundedness of nonautonomous superposition operators in BV[0,1], Bull. Aust. Math. Soc. 92 (2015), 325-341.

[18] Y. KIM AND L.A. VESE, Image recovery using functions of bounded variation and Sobolev spaces of negative differentiability, Inverse Probl. Imag., 3 (2009), 43-68.

[19] A.G. LJAmin, On the acting problem for the Nemytskij operator in the space of functions of bounded variation, $11^{\text {th }}$ School Theory Oper. Function Spaces, Chel'jabinsk (1986), 63-63 (in Russian).

[20] S. Łojasiewicz, An Introduction to the Theory of Real Functions, John Wiley \& Sons, Ltd., Chichester, 1988.

[21] V. MAZ'YA, Sobolev Spaces with Applications to Elliptic Partial Differential Equations, Springer, Grundlehren der Mathematischen Wissenschaften, vol. 342, 2011.

[22] P. Má́Kowiak, A counterexample to Ljamin's theorem, Proc. Amer. Math. Soc., 142 (2014), 1773-1776.

[23] A. Piskorek, Równania Catkowe (Integral Equations), Wydawnictwa NaukowoTechniczne, Warszawa, 1980 (in Polish). 
[24] D. Waterman, On $\Lambda$-bounded variation, Studia Math. 57 (1976), 33-45.

\section{Manuscript received September 22, 2015}

accepted January 26, 2016

Daria Bugajewska, Dariusz Bugajewski and Piotr Kasprzak

Optimization and Control Theory Department

Faculty of Mathematics and Computer Science

Adam Mickiewicz University

ul. Umultowska 87

61-614 Poznań, POLAND

E-mail address: dbw@amu.edu.pl,ddbb@amu.edu.pl, kasp@amu.edu.pl

Piotr MaćKOWiak

Department of Mathematical Economics

Poznań University of Economics

Al. Niepodległości 10

61-875 Poznań, POLAND

E-mail address: p.mackowiak@ue.poznan.pl 